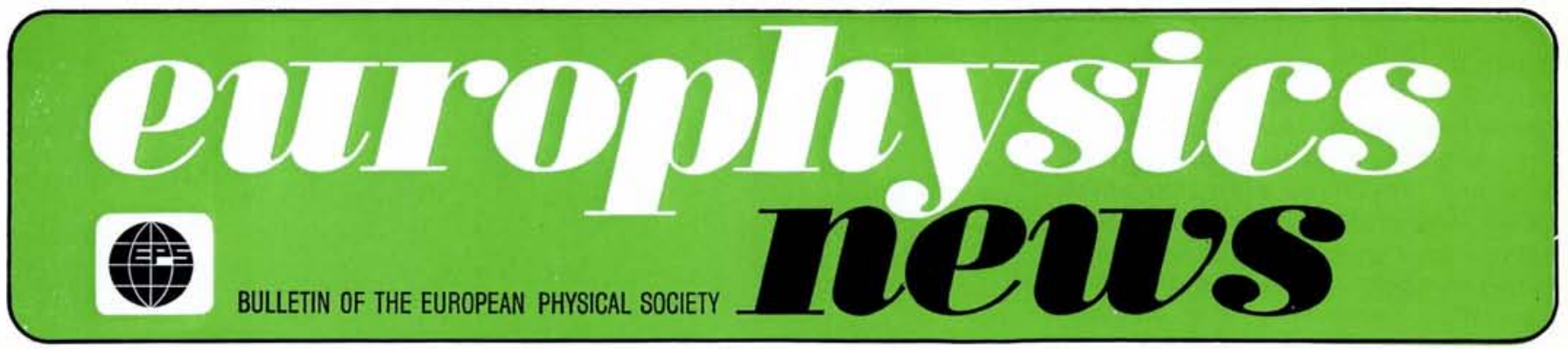

On the occasion of the EPS Council meeting, held on 28-29 March in Geneva, J. Friedel, Orsay had been asked to give a scientific talk to the participants. The text of his lecture is reprinted here for the readers of Europhysics News.

The subject of my talk might seem somewhat narrow or even esoteric, compared with many topics of perhaps more fundamental interest or of more direct applications that I could have chosen in the field of condensed matter. However it shows how simple concepts can help in building a useful and unifying language, to analyse rather complex situations. This is a general attitude in our field; and it is especially comforting to see how european physicists, chemists, metallurgists and biologists have joined their efforts here, and provided most of our knowledge and of our ideas in what is a truly interdisciplinary field.

\section{Dislocations in Solids}

Interested in prestressed concrete, Volterra showed that any state of internal stress in a continuous solid can be described as due to a distribution of lines of discontinuity of these stresses, or 'dislocations'.

Each can be thought of as produced by the Volterra process:

- Cut a piece of unstressed solid along a surface $S$ bordered by the line $\mathrm{L}$.

- Displace the lips of the cut with respect to each other without changing their form. This usually produces a gap or matter in excess.

- Fill in the gap or remove the matter in excess, and stick the two lips together again.

- Remove all the external stresses that were applied during the process.

One is left with internal stresses that are obviously discontinuous along L. They are continuous across S, because stresses are proportional to distortions, and no discontinuity of distortion has been introduced along $S$ by the process.

\title{
Dislocations in Liquid Crystals
}

\section{J. Friedel, Orsay}

The dislocation line is then defined by its geometrical position L and by its strength, the relative displacement. There is obviously a conservation theorem, which states that when a dislocation splits into two others, their relative displacements must add to the relative displacement of the initial one.

Any displacement can be thought of as the sum of a translation and a rotation. Simple geometry then shows that a translation dislocation with a translation (or 'Burgers vector') b can split into two translation dislocations $\vec{b}$ and $\overrightarrow{b^{\prime \prime}}$ such that $\vec{b}=\overrightarrow{b^{\prime}}+\overrightarrow{b^{\prime \prime}}$ (Fig. 1.a); it can also split into two rotation dislocations of parallel axes and opposite strength $\pm \Omega$, at a distance $d$ related to $b$ and $\Omega$ (Fig. 1.b). Conversely, a rotation dislocation can split into a rotation dislocation of same strength and parallel axis and a translation dislocation (Fig 1.c). Finally a rotation dislocation is equivalent to a continuous distribution of infinitesimal translation dislocations: the sector of angle $\Omega$ which is intro-

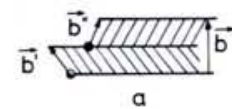

a
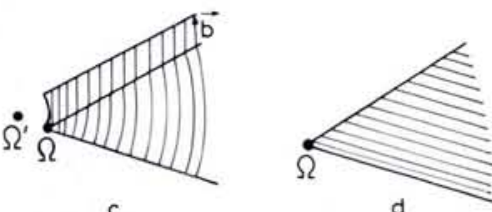

Fig. 1. Splitting of dislocations, a : translation dislocation into two trans/ation dislocations; $b$ translation dislocation into two rotation dislocations : c: rotation dislocation into a rotation dislocation and a translation dislocation: $d$ rotation dislocation as a continuous distribution of infinitesimal translation dislocations. duced when the rotation dislocation is created is equivalent to a pack of thin slices of constant thickness (Fig. 1.d). As a result of this last remark, translation dislocations are sufficient to describe any state of internal stress in a continuous solid.

In a crystal, the possible displacements are limited by the fact that, at the end of the Volterra process, the two lips of the cut must be in phase to be able to stick without leaving a fault along the cut. The displacements are 'quantized': they must be symmetry operations of the crystal.

Translation dislocations have then

Burgers vectors $\vec{b}$ equal to periods of the 'Bravais lattice' of the crystal. Their long range strains are propor-

tional to $\vec{b}$, thus lead to a long range elastic energy proportional to $b^{2}$, and little dependent on the position of the line. Dislocations with multiple periods have then a tendency to split into dislocations with elementary periods, so as to minimize the energy, following Pythagore's theorem. By moving, a dislocation line $L$ extends the surface $S$ of the cut. This usually requires adding or removing matter along $L$; this is possible at high temperatures where self diffusion takes place. At low temperatures, motion of the trans-

\section{Contents}

Dislocations in Liquid Crystals Letter to the Editor

Society News

One-dimensional Electron Systems

The 2nd Europhysics Conference of the Section of Macromolecular Physics

Meetings .
1 5 5 6 8 
lation dislocations remains possible parallel to their Burgers vector, because this does not open a gap along $\mathrm{S}$; it is easy, at least in most metallic and ionic structures when the short range strains in the core of the dislocations have an energy which remains fairly constant during motion. Only translation dislocations with elementary periods are therefore present in most crystals. Their existence was invoqued between the two wars by Orowan, Polanyi and Taylor to explain the plasticity of crystals. But it is only after the last war that they were observed and studied, mostly under the impetus of the british school.

Rotation dislocations have an energy which varies with position, and is a minimum when the line lies on the axis of rotation. Their motion then involves enormous stresses: they are 'sessile'. Their energy is also proportional to the square of a critical distance which can be that to the outside surface or to a parallel rotation dislocation of opposite sign. As a result, these dislocations are unlikely to be formed in crystals, except for exceptional cases (Se or Te crystals, lattice of vortex lines in supraconductors of the second kind) where some translation dislocations can split into close pairs of opposite rotation dislocations. They are a mere curiosity in crystals.

\section{Liquid Crystals}

They are fluids with anisotropic properties. First observed by a biologist Reinitzer (1888), they were much studied by his collegue Lehman and classified by $\mathrm{G}$. Friedel according to their molecular arrangement. They are all built by a packing of elongated molecules with a general formula

$$
X-\longrightarrow Y-\longrightarrow
$$

The rigid body contains in $\mathrm{Y}$ : $-\mathrm{NO}=\mathrm{N}-,-\mathrm{N}=\mathrm{N}-$ or $-\mathrm{CO}-\mathrm{O}-$. The flexible tails are made in $\mathrm{X}$ and $\mathrm{Z}$ by $-\mathrm{O}-\mathrm{C}_{\mathrm{m}} \mathrm{H}_{2 \mathrm{~m}+1}$ or $-\mathrm{C}_{\mathrm{n}} \mathrm{H}_{2 \mathrm{n}+1}$.

In the nematic phases, the centers of gravity of the molecules are arranged more or less at random, except for a short range order and they fluctuate rapidly in position, as in an isotropic liquid. But the axes of the molecules have an average direction, which is well defined in a macroscopic region. There are equal numbers of molecules pointing in the positive ( $X Y Z$ ) and in the negative ( $Z Y X)$ directions (Fig. 2.a).

Cholesteric phases can be thought of as deduced from a nematic phase by a static torsion with an axis perpendicular to the molecules. This helical arrangement is obviously related
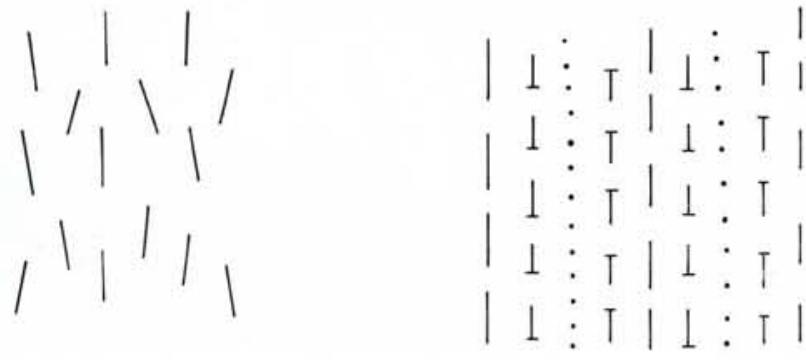

||||||$/||||$ $11 \| 1111111$

$|/||/||/ / \||$ | ||||$/|| /||$ b

c

Fig. 2. Mesomorphic phases in liquid crystals : $a$ : nematic ; b : cholesteric ; $c:$ smectic $A$.

to some skew character of the molecules. Again, as XYZ and ZYX molecules are randomly piled together, the helical arrangement has a period equal to half its pitch (Fig. 2.b).

In smectic phases, molecules are piled parallel to each other in successive equidistant planes. In the smectic A phases for instance, molecules are normal to the planes and have no long range order within the plane (Fig. 2.c).

Interest in these structures has been revived because of the small energies involved in their ordering. They are indeed equilibrium phases ('mesomorphic' phases), with well defined latent heats of transition. But these are very small and their structure is easily perturbed. Indeed any kind of perturbation one can think of has been tried: temperature, thermal gradient, ac and dc electric field and electric current, magnetic field, flow or boundary conditions. From a practical point of view, three main effects have been studied: the variation of pitch in cholesterics; the onset of convective flow in nematics or cholesterics; bending modes in smectics. All have characteristic lengths which can be comparable with optical wave lengths; they involve large changes in molecular orientations and are re-

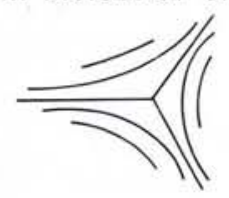

$-\pi$

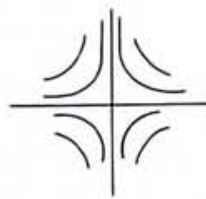

$-2 \pi$

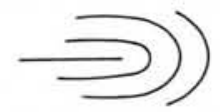

$+\pi$
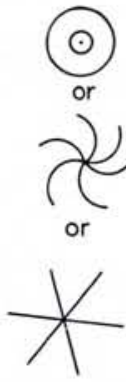

$+2 \pi$
Fig. 3. Rotation dislocations in nematics. laxed by a viscous flow of the molecules. In a nematic phase for instance, the three types of possible static distortions all correspond to progressive rotations of the molecules from their initial orientation, with no dilatation: the 'torsion' mode is that observed spontaneously in cholesterics (Fig. 2.b); in the 'splay' mode, there is a fanning out of molecular orientations; in the 'bending' mode, the lines defined by the molecular orientations have a general curvature.

\section{Dislocations in Liquid Crystals}

A direct study can be made of possible singularities in these static distortions. The names of Kröner, de Wit and more especially Kleman and $\mathrm{Na}$ barro are associated with this effort. It is more instructive to ask first what are the possible dislocations obtained by the Volterra process in a solid with the same symmetry properties as a liquid crystal. One then can see how these are changed by viscous relaxation when going to the real liquid crystal. The first question is not an empty one, as one can now, following a suggestion by de Gennes, solidify a liquid crystal by polymerisation; also some biological materials are solids with liquid crystal symmetry.

\section{a) Nematic phases}

In a solid with nematic symmetry, the Volterra process can produce two types of dislocations:

- Quantized dislocations, where the axis of rotation is perpendicular to the molecules and the rotation is a multiple of $\pi$. Figure 3 shows the arrangements of molecules for dislocations perpendicular to the plane of figure and rotations of $\pm \pi, \pm 2 \pi\left(S= \pm \frac{1}{2}, \pm 1\right.$ in the accepted notation). For $\Omega=+2 \pi$, different orientations of the surface of cut with respect to the orientation of the molecules lead to different configurations.

- Non quantized dislocations, corresponding to any translation or to any rotation with an axis parallel to the molecules. 
When now such a solid melts into a liquid crystal, all translation dislocations can move quickly by diffusion and split into dislocations of smaller Burgers vectors. They quickly disperse so that all their elastic energy disappears. Rotation dislocations can bend and move by emitting such translation dislocations, of no energy. Non quantized rotation dislocations therefore also split and disperse. Quantized rotation dislocations annihilate each other or at the surface, except for a few that remain and are stabilized by suitable boundary conditions (irregularities on the surface...). These remaining ones relax most of their energy by attracting a cloud of infinitesimal translation dislocations, in such a way that only torsion, bend or splay distortions remain.

Because they strongly distort the structure, such few defects should give a strong optical contrast and be clearly visible under the microscope. Indeed their presence is so characteristic of the nematic phases that they gave them their name (nema, nemata $=$ thread in greek). These dislocations lines were observed by Lehman; their topology was understood by G. Friedel in 1920, thus 30 years before dislocations were observed in solid crystals. The optical contrast obtained from Frank's analysis of the strains, Fig. 3 , is in good agreement with observation for $-\pi$ dislocations. A slight complication is connected with the fact that dislocations of strength $\pm 2 \pi$ are observed, although, with the structure of Fig. 3, they should split into dislocations of strenth $\pm \pi$. What happens has been shown by the following experiment, performed in Orsay by Cladis, Williams and Kleman: a nematic liquid is introduced in a capillary tube, treated in such a way that the molecules must arrive normal to the tube. One then expects a dislocation of strength $+2 \pi$ along the axis of the tube. However the molecules bend away from their planar arrangements so as to remove the line of discontinuity (Fig. 4.a). Singularity points only occur at both ends in the tube, and possibly at some regions in the tube where the bending changes sign (Fig. 4.b). The total defect is still topologically equivalent to a $+2 \pi$ dislocation; it has a fuzzier optical contrast, and a definitely reduced line tension, which makes it stable with respect to splitting into two $+\pi$ dislocations. Indeed the same type of relaxation can occur for $-2 \pi$ dislocations, but not for $\pm \pi$ ones. Singularity points were actually first studied by Meyer from Harvard in a different geometrical arrangement.
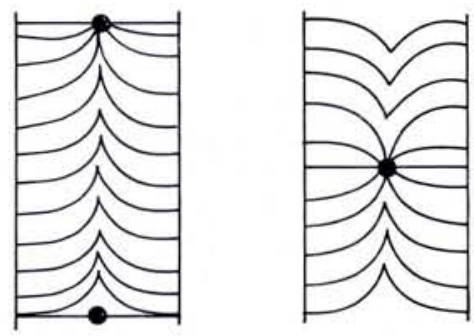

a

b

Fig. 4. Singularity points on a split $2 \pi$ disiocation in a nematic.

There is a strong analogy of this field with that of discontinuity lines in ferromagnetics. The main difference is that magnetization is given at each point by its direction and its sign, thus by a vector field and not by a director field as in nematics. Only rotation dislocations $\pm 2 \pi$ are then expected, with the same relaxation leading to continuous cores except for singular points. The magnetization has usually domains of preferred orientation, due to magnetoelastic or magnetocrystalline effects: this is similar to a nematic under an applied magnetic or electric field which also tends to align the molecules in a given direction. The 'crossties' configurations observed in such thin magnetic films are alternate $\pm 2 \pi$ dislocations along domain walls. The analogue of nematics without external perturbation would be stress free amorphous ferromagnetics without long range stresses; here too the boundary conditions would regulate the presence of $\pm 2 \pi$ dislocations and of singular points.

There are also some partial analogies with vortex lines in suprafluids.

\section{b) Smectic phases}

The close packing of the planes suggests that their bending is much easier than a change in their equidistance. If the equidistance is strictly kept, one obtains a set of equidistant surfaces, with common normals. Their envelope is usually a surface $\Sigma$ into which the structure cannot penetrate. To have a structure filling a whole region in space, one must force the 'developing' surface $\Sigma$ to reduce to a set of lines. A standard geometry result is that these lines are 'focal conics', i.e. an ellipse and a branch of hyperbola with one common axis, lying in orthogonal planes, each passing by a focus of the other. The equidistant surfaces are then 'Dupin cyclides', with normals touching both lines. Particular cases are concentric spheres or coaxial cylinders. The focal conics are lines of discontinuity

\section{ELECTRON AND PHOTON \\ INTERACTIONS AT HIGH ENERGIES}

Proceedings of the 6th International Symposium, Physikalisches Institut, University of Bonn, August $27 \cdot 31,1973$

edited by H. ROLLNIK and W. PFEIL

\section{4. about 566 pages. US\$73.10/Dfl. 190.00}

These Symposia are organized biennially under the auspices of the International Union of Pure and Applied Physics. This series of conferences deals with the interaction of electrons and photons with themselves and with other particles, such as hadrons. At the 1973 Symposium, the interaction of high energetic neutrino beams with matter was introduced as a topic for discussion.

In 8 plenary sessions the following topics have been presented and discussed:

Electron Storage Ring Physics; Photoand Electroproduction in the Resonance Region; Exclusive and Inclusive

Reactions induced by Electromagnetic Currents; Neutrino Reactions at High Energies; Currents and Fields; Lepton's Future.

\section{ELECTROMAGNETIC EXCITATION}

Theory of Coulumb Excitation with Heavy Ions

by $\mathrm{K}$. ALDER and A. WINTHER

1974. about 368 pages.

US\$42.30/Dfl. 110.00

This present monograph aims at presenting an exhaustive description of the non-relativistic theory of excitations in heavy-ion nuclear collisions caused by the electromagnetic field. Tables and graphs are given for the analysis of experiments and the text is illustrated by selected experimental results.

The presentation is based on semiclassical methods, which find aplication also for other atomic and nuclear processes.

\section{NORTH-HOLLAND PUBLISHING CO.}

P.O. Box 211

AMSTERDAM,

The Netherlands

Sole distributors for the U.S.A. and Canada

American Elsevier

Publishing Company

52 Vanderbilt Avenue

NEW YORK, N.Y. 10017 
topologically equivalent to $+2 \pi$ rotation dislocations.

It is clear that such a texture cannot alone fill, in general, a given space with given boundary conditions. Nature must pile such domains together, and fill the interstices with more and smaller such domains. This is the texture usually met in smectic phases i.e. in soaps, and also in related layered polymeric phases.

A closer inspection shows that neighbouring focal conics domains sometimes meet at an angle, so as to fill the interstice between them. The line of contact between three such distorted domains would be a $-\pi$ dislocation. Also translation dislocations analyzed theoretically by de Gennes, Pershan and Kleman, have recently been observed in Orsay, both in smectics and in lipidic membranes of related structure. These deviations from the perfect Duplin cyclides arrangement are made possible by a small variation in the equidistance between successive planes. The core structure and mobility of these translation dislocations are under study. The possibilities of relaxation are much more restricted than in nematics, and the whole texture is more stable and difficult to alter.

c) Cholesteric phases

They are layered structures with an equidistance more easy to alter than in smectics. As a result, one usually observes in perturbed cholesteric structures very complex textures, which are a mixture of focal conic domains and of distorted regions full of dislocations. These have been much studied in recent years, especially by Bouligand, and as pointed out by him and others, are analogous to textures observed in biological materials with local cholesteric arrangement.

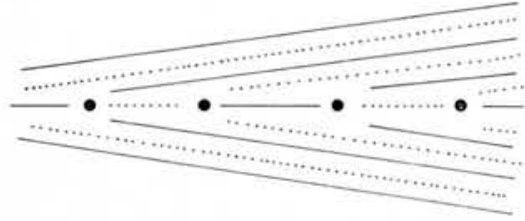

Fig. 5 Grandjean's experiment with translation dislocations.

I shall restrict myself to simpler cases of nearly perfect cholesteric structure with a few dislocations.

The first case is that of a layer of cholesteric between two glass plates which have been rubbed with a greasy finger, so as to force the molecules touching the plates to lie parallel to the plates in a given direction. If the two plates are not exactly parallel, there will be regions where the thickness is near to an integral number

\section{Tf UNIUERSTTY OF NIJMEGEN 1 THE NETHERLANDS}

In the Institute of Theoretical Physics at the University of Nijmegen, the Netherlands, is a position open for a

\section{lector of theoretical physics}

\section{A lectorposition corresponds roughly with an associate professorship.}

Applicants should be theorists versed in statistical physics and with an active interest in solid state physics.

Applications (including curriculum vitae and list of publications) should be addressed to:

Prof. Dr. A. G. M. Janner,

Instituut voor Theoretische Natuurkunde,

Toernooiveld, Nijmegen. The Netherlands.

Suggestions concerning suitable condidates are also welcome.

of half pitches, where the structure will be little perturbed. These regions will be separated by regions where the number of half pitches varies abruptly by one unit, in a kind of Vernier effect. It was realized already in 1930 that most of the cholesteric planes should be continuous across each of these walls, except for a small region of large distortions along a line (Fig. 5). Such equidistant lines indeed show up optically. They are obviously translation dislocations with strength equal to half a pitch.

Careful observation shows however that in thick samples these dislocations are replaced by fuzzier dislocations with a Burgers vector equal to a full pitch. The reason is that such dislocations can split into two rotation dislocations with a split core and no line singularity (Fig. 6), a structure which has been studied in detail by Bouligand and Strzelecki in cholesterics with large pitch, polymerized and sliced for examination. Such stabilizing splitting cannot occur in thin samples, where molecules are forced by the boundary conditions to keep within their cholesteric planes.

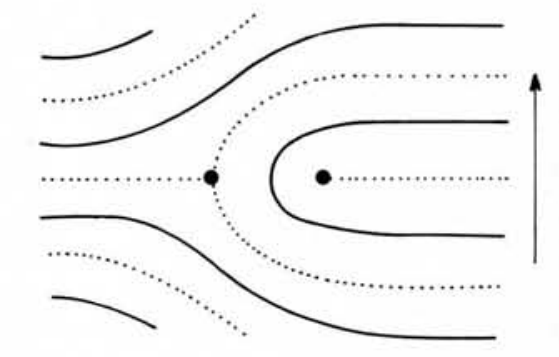

Fig. 6. Translation dislocation split into two rotation dislocations in a cholesteric.

Such a split dislocation can actually bend without much expense of energy if, while bending, it moves parallel to the torsion axis of the cholesteric, so as to be always parallel to the molecules of its cholesteric plane. Successive turns thus produce a very stable helical arrangement of the dislocation. Helical dislocations, with a pitch parallel to the general cholesteric pitch, are often observed; they have been studied by Rault, Bouligand and Kleman. As each turn of the helical dislocation introduces a supplementary torsion on one side of the dislocation, two extreme configurations can thus be obtained:

- a cholesteric structure, with a nematic structure or other matter inside the helix. This may be the description of a pore in the skin of some crabs, where the constituting elongated molecules have a cholesteric arrangement;

- a stick of cholesteric matter folded within a helix and surrounded by 
other matter. This might help to describe the folding of DNA molecules in some large chromosomes with cholesteric structure. Further $(-\pi)$ dislocations seem involved in the splitting of these chromosomes.

\section{Conclusion}

I heave tried to give a feeling about a part of condensed matter research which deals with intermediary structures, between the macroscopic and the really microscopic one. This has been an active field for some time, well known in metallurgy, magnetism, and electronics. It seems also a promising field at the boundary of polymer physics and of biology.

\section{Society News}

\section{Member Societies}

The Institute of Physics, London announces that A.B. Pippard, Cambridge took over the presidency of the Institute from Sir Brian Flowers.

\section{Associate Members}

The following organizations have decided to join the European Physical Society as Associate Members :

Agip Nucleare S.p.A., Milano, Italy, Manufacture Belge de Lampes et de Matériel Electronique S.A., Bruxelles, Belgium.

\section{Improving on the Physics Society Interaction}

G. Diemer, Chairman of the Advisory Committee on Physics and Society points out that the letter to member societies which forms the main part of the text published under the above title in Europhysics News, 5, No. 6 (June 1974) was actually signed by H.B.G. Casimir, President.

\section{Europhysics Journals}

Upon recommendation of the Advisory Committee on Publications the Executive Committee granted the EPS label to Contemporary Physics, a review journal.

Optica Acta recently accepted as specialized Europhysics journal will be available to Individual Ordinary Members of EPS at the reduced rate of $£ 12$ per annum, provided that individuals warrant the Journal for their personal use. Orders may be placed directly to the Publisher, Taylor \& Francis, 10-14 Macklin Street, London WC2B 5NF.

\section{Letter \\ to the Editor}

Sir,

The letter to the Editor by B.J. Green in EUROPHYSICS NEWS 5, No. 3 (1974) cought my interest. I am one of those European physicists who have not yet felt enough urge to enlist as "individual ordinary member " of EPS. And this in spite of the fact that I live closest to EPS headquarters and know its Geneva staff personally; in spite also of the fact that EUROPHYSICS NEWS is printed by the publisher of my local newspaper.

Being an active member of one of the member societies of EPS and Physics professor at a European University my interest in European Physics is obvious. But, while I had been enthousiastic about the founding of EPS, the final product left me somewhat embarrassed, in particular when comparing with the American Physical Society, of which I am also a member: The compromising about EPS journals was a sad start, and I came to admit that Helvetica Physica Acta was wise in not buying the EPS seal. Unfortunately, I must agree with Green on this point.

As to the question of the longrange policy matter raised in Green's letter I would like to remind you of the article by K. Ganzhorn in EUROPHYSICS NEWS 3, No. 1 (1972). In view of this courageous and realistic appreciation of the problem the "Note from the Editor" following Green's letter sounds deceptively lame.

I think Ganzhorn has really put the finger on one of the problems of EPS when he writes "All indicated tasks in science policy are of a nature to defy solution by honorary engagement". It seems, indeed, to be a general feature of Science policy patterns in
Europe as compared to the United States, where scientists are appointed to serve in political bodies on a fulltime basis over a restricted number of years, that in Europe these appointments are often on a honorary or part-time basis extending over long periods. Here the example best known to me is the Swiss National Fund.

The trouble is that the problem of the honorary engagement is intimately coupled to the financial situation of EPS which by itself reflects an unrealistic policy followed in the past, and which now apparently excludes all but honorary engagements.

This pattern looks rather like being self-trapping. The only way out I can see at this moment is that strongly motivated European scientists devote their sabbatical years to Science policy jobs with EPS. There is of course also the honorable example of the acting president of EPS who serves while being retired. But there are several obvious reasons that this example hardly can be generalised.

I think that in the long run EPS should arrive at an integration of national societies in such a way that the distinction between "individual ordinary members " and national society members would vanish. In fact this distinction gives rise to the image of a split social personality of EPS. And this leads to another self-trapping mechanism: It makes it difficult for individual physicists to identify with EPS and hence reduces the attractiveness to enlist as « individual ordinary member $"$.

Once EPS would have found its " unsplit " personality in the above sense the financial strengthening would follow rather naturally and the problem of the EPS journals could be settled more forcefully. Needless to say that such a unification would also lead to a strengthened and better motivated leadership of EPS.

\section{C.P. Enz,}

Geneva University

\section{Divisions}

The following Sections of the Condensed Matter Division recently elected their new Boards. A Libchaber, Secretary of the Division reports that the following have been elected
LOW TEMPERATURE PHYSICS :

W.J. Huiskamp, Leiden W. Klose, Saarbrücken N. Kurti, Oxford J. Lindqvist, Uppsal $\mathrm{S}$. Safrata, Rez near Prague METALS :

P. Averbuch, Grenoble O. Beckman, Uppsala F. Fumi, Genoa V. Heine, Cambridge J. Muller, Geneva w. Schilling, Jülich

\section{MACROMOLECULAR \\ MAGNETISM} PHYSICS

P. Corradini, Naples E.W. Fischer, Mainz W. Holzmüller, Leipzig A.J. Kovacs, Strasbourg I.M. Ward, Leeds
A.S. Borovik-Romanov, Moscow G. Busch, Zürich

A.R. Miedema, Eindhoven

R. Pal, Budapest

E.P. Wohlfarth, London
SEMICONDUCTORS AND INSULATORS :

G. Baldini, Milan

M. Balkanski, Paris

F. Bassani, Rome

P. Grosse, Aachen

c. Hilsum, Great Malvern

B.M. Vul, Moscow 